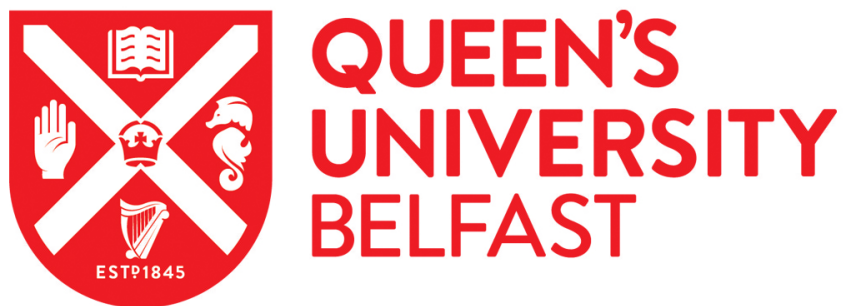

\section{Molecular line emission from a protoplanetary disk irradiated externally by a nearby massive star}

Walsh, C., Millar, T. J., \& Nomura, H. (2013). Molecular line emission from a protoplanetary disk irradiated externally by a nearby massive star. Astrophysical Journal Letters, 766(2), [L23]. https://doi.org/10.1088/20418205/766/2/L23

Published in:

Astrophysical Journal Letters

Document Version:

Publisher's PDF, also known as Version of record

Queen's University Belfast - Research Portal:

Link to publication record in Queen's University Belfast Research Portal

Publisher rights

(c) 2013 IOP Publishing

\section{General rights}

Copyright for the publications made accessible via the Queen's University Belfast Research Portal is retained by the author(s) and / or other copyright owners and it is a condition of accessing these publications that users recognise and abide by the legal requirements associated with these rights.

Take down policy

The Research Portal is Queen's institutional repository that provides access to Queen's research output. Every effort has been made to ensure that content in the Research Portal does not infringe any person's rights, or applicable UK laws. If you discover content in the Research Portal that you believe breaches copyright or violates any law, please contact openaccess@qub.ac.uk. 


\title{
MOLECULAR LINE EMISSION FROM A PROTOPLANETARY DISK IRRADIATED EXTERNALLY BY A NEARBY MASSIVE STAR
}

\author{
Catherine Walsh ${ }^{1,2}$, T. J. Millar ${ }^{1}$, and Hideko Nomura ${ }^{3}$ \\ ${ }^{1}$ Astrophysics Research Centre, School of Mathematics and Physics, Queen's University Belfast, University Road, \\ Belfast BT7 1NN, UK; cwalsh@strw.leidenuniv.nl \\ ${ }^{2}$ Leiden Observatory, Leiden University, P.O. Box 9513, NL-2300 RA Leiden, The Netherlands \\ ${ }^{3}$ Department of Astronomy, Graduate School of Science, Kyoto University, Kyoto 606-8502, Japan \\ Received 2012 December 28; accepted 2013 March 4; published 2013 March 19
}

\begin{abstract}
Star formation often occurs within or nearby stellar clusters. Irradiation by nearby massive stars can photoevaporate protoplanetary disks around young stars (so-called proplyds) which raises questions regarding the ability of planet formation to take place in these environments. We investigate the two-dimensional physical and chemical structure of a protoplanetary disk surrounding a low-mass (T Tauri) star which is irradiated by a nearby massive O-type star to determine the survivability and observability of molecules in proplyds. Compared with an isolated star-disk system, the gas temperature ranges from a factor of a few (in the disk midplane) to around two orders of magnitude (in the disk surface) higher in the irradiated disk. Although the UV flux in the outer disk, in particular, is several orders of magnitude higher, the surface density of the disk is sufficient for effective shielding of the disk midplane so that the disk remains predominantly molecular in nature. We also find that non-volatile molecules, such as $\mathrm{HCN}$ and $\mathrm{H}_{2} \mathrm{O}$, are able to freeze out onto dust grains in the disk midplane so that the formation of icy planetesimals, e.g., comets, may also be possible in proplyds. We have calculated the molecular line emission from the disk assuming LTE and determined that multiple transitions of atomic carbon, $\mathrm{CO}$ (and isotopologues, ${ }^{13} \mathrm{CO}$ and $\mathrm{C}^{18} \mathrm{O}$ ), $\mathrm{HCO}^{+}$, $\mathrm{CN}$, and $\mathrm{HCN}$ may be observable with ALMA, allowing characterization of the gas column density, temperature, and optical depth in proplyds at the distance of Orion $(\approx 400 \mathrm{pc})$.
\end{abstract}

Key words: astrochemistry - line: profiles - protoplanetary disks - stars: formation

Online-only material: color figures

\section{INTRODUCTION}

Star formation rarely takes place in isolation; stars form primarily within multiple systems and often within, or nearby, massive stellar clusters (see, e.g., Kennicutt \& Evans 2012). Thus, the young star's protoplanetary disk can be heavily irradiated and indeed, photoevaporated, by nearby massive stars (see, e.g., Bally et al. 1998), for example, the famous proplyds in the Orion nebula (O'Dell et al. 1993). The properties of proplyds have been well studied since their discovery; however, little is known about their molecular content due to their small size $(R \sim 100-1000 \mathrm{AU})$ and relatively large distance, e.g., Orion at $\approx 400 \mathrm{pc}$. Protoplanetary disks are the sites of planet formation and the study of disks in extreme environments is important for a complete understanding of this process. Can the disk survive sufficiently long for planet formation to take place? This has implications on our understanding of the formation of our own solar system as it is thought that the Sun formed within a stellar cluster which has long since dispersed (see, e.g., Adams 2010 and references therein).

We calculate the chemical structure and molecular line emission from a protoplanetary disk around a classical T Tauri star which is irradiated by a nearby $(\lesssim 0.1 \mathrm{pc})$ massive O-type star. Our aims are (1) to determine whether molecules are able to form and survive in the disk, (2) to identify species that may be observable with ALMA, and (3) to identify species that can characterize the physical conditions and the subsequent implications on planet formation.

\section{PROTOPLANETARY DISK MODEL}

\subsection{Physical Model}

We model a steady axisymmetric disk in Keplerian rotation about a classical T Tauri star with a mass of $0.5 M_{\odot}$, a radius of $2 R_{\odot}$, an effective temperature of $4000 \mathrm{~K}$, and $\alpha=0.01$, using the methods outlined in Nomura \& Millar (2005) and Nomura et al. (2007). The radial surface density distribution is determined assuming a constant mass accretion rate, $\dot{M}=$ $10^{-8} M_{\odot} \mathrm{yr}^{-1}$ (Pringle 1981). To determine the density and temperature structure, we solve the equation of hydrostatic equilibrium in the vertical direction and the local thermal balance between the heating and cooling of the gas. We include grain photoelectric heating by UV photons and heating via hydrogen ionization by $\mathrm{X}$-rays and allow the gas to cool via gas-grain collisions and line transitions.

Our disk is irradiated by UV photons and X-rays from the central star and UV photons from the interstellar radiation field and a nearby massive O-type star. We model the UV spectrum of the O-type star as a blackbody with an effective temperature of $45,000 \mathrm{~K}$. We set the UV flux at the disk surface to $\left(4 \times 10^{5}\right) \times$ $G_{0}$, where $G_{0} \approx 1.6 \times 10^{-3} \mathrm{erg} \mathrm{cm}^{-2} \mathrm{~s}^{-1}$ is the average strength of the integrated interstellar radiation field. This corresponds to a distance between the disk and the massive star of $\lesssim 0.1 \mathrm{pc}$.

We assume the dust and the gas in the disk are well mixed and adopt a dust-grain size distribution which reproduces the extinction curve observed in dense clouds (Weingartner \& Draine 2001). We note that this is a simplistic treatment of 
the dust distribution in disks since it is thought that gravitational dust settling and coagulation perturb the dust size and density distribution from that observed in dense clouds (see, e.g., Dullemond \& Dominik 2004; D'Alessio et al. 2006).

\subsection{Chemical Model}

We use the gas-grain chemical network from Walsh et al. (2010) and compute the photoreaction rates and X-ray ionization rates using the methods outlined in Walsh et al. (2012). Our gas-phase chemistry is from the UMIST Database for Astrochemistry (Woodall et al. 2007). We include freezeout of gasphase material onto dust grains and thermal and non-thermal desorption. We allow photodesorption by both external and internal UV photons and desorption via cosmic-ray heating of dust grains (Hasegawa \& Herbst 1993; Öberg et al. 2009a, 2009b). We use a small grain-surface network to include the synthesis of several complex organic molecules, e.g., methanol (Hasegawa et al. 1992; Hasegawa \& Herbst 1993). We include the photodestruction of grain mantle material by UV photons, X-rays, and cosmic rays (Ruffle \& Herbst 2001). Experiments have shown that the irradiation of interstellar ice analogs by UV and $\mathrm{X}$-ray photons induces chemistry in the ice (see, e.g., Hagen et al. 1979; D'Hendecourt et al. 1986; Allamandola et al. 1988; Öberg et al. 2010b; Ciaravella et al. 2010). We adopt the equivalent gas-phase photorates for ice species and allow the products to remain on the grain to take part in further grain-surface reactions.

We assume the grains are negatively charged compact spheres with a radius of $0.1 \mu \mathrm{m}$ and a constant fractional abundance of $\sim 10^{-12}$ relative to the gas number density, equivalent to a gasto-dust mass ratio of $\sim 100$. We adopt a sticking coefficient, $S \approx 1$, for all species. We include the dissociative recombination of gas-phase cations with dust grains. The dust model used for the chemistry is simple compared with that used to calculate the disk physical structure; however, the total dust-grain cross section is consistent between models.

Our initial abundances are from the output of a dark cloud model at an age of $\sim 10^{5}$ yr. Our underlying elemental abundance ratios for $\mathrm{H}: \mathrm{He}: \mathrm{C}: \mathrm{N}: \mathrm{O}: \mathrm{Na}: \mathrm{Mg}: \mathrm{Si}: \mathrm{S}: \mathrm{Cl}: \mathrm{Fe}$ are 1.00:0.14:7.30(-5):2.14(-5):1.76(-4):3.00(-9):3.00(-9):3.00 $(-9): 2.00(-8): 3.00(-9): 3.00(-9)$. We map the chemical structure of the disk at a time of $10^{6} \mathrm{yr}$, the typical age of classical $\mathrm{T}$ Tauri stars.

\subsection{Molecular Line Emission}

To estimate the molecular line emission, we assume the disk is "face-on" and that local thermodynamic equilibrium (LTE) holds throughout. We calculate the emergent flux from the top (i.e., visible) half of the disk only which we also assume is the surface directly irradiated by the nearby massive star. In reality, the lower half, although not directly irradiated by the nearby star, is subject to diffuse, scattered UV radiation originating from the stellar cluster, and this needs to be taken into account in the calculation of the physical structure of the lower disk. Hence, our estimated molecular line emission strengths are lower limits to the potentially observable line strengths. We include only thermal broadening of the line profiles because this will dominate turbulent broadening. For $\mathrm{CO}$ isotopologues, we assume the molecular isotopic ratios reflect the atomic ratios and use values applicable for the local interstellar medium: ${ }^{13} \mathrm{C} /{ }^{12} \mathrm{C}=1 / 77$ and ${ }^{18} \mathrm{O} /{ }^{16} \mathrm{O}=1 / 560$ (Wilson \& Rood 1994).

\section{RESULTS}

\subsection{Physical Structure}

In Figure 1, we present the gas temperature, number density, integrated UV flux, and integrated X-ray flux as a function of disk radius, $R$, and height, $Z / R$. We also display these parameters, along with the cosmic-ray and X-ray ionization rates, as a function of height at $R=100$ AU. We compare results from our irradiated disk (solid lines) and isolated disk (dashed lines; see Walsh et al. 2012). Here, "irradiated" refers to a disk irradiated by a nearby massive star and the central star, whereas "isolated" refers to a disk irradiated by the central star only.

Compared with the isolated disk, the gas and dust temperatures in the irradiated disk are significantly higher in the outer disk midplane $(R \gtrsim 1 \mathrm{AU})$. The gas temperature structure in the innermost region, $R \lesssim 1 \mathrm{AU}$, is similar in both disks because this is driven by irradiation by the central star. The density structure is also perturbed, depending on the gas temperature profile.

In the surface at $100 \mathrm{AU}$, we see around an order of magnitude decrease in the X-ray flux and subsequent ionization rate in the irradiated model, compared with the isolated model. This is due to the differing attenuation of X-rays along the line of sight from the central star, which is affected by the density and gas temperature profile in the disk. In the disk midplane, the X-ray flux (and ionization rate) is similar in both models. We see a large increase in the UV flux in the disk surface, increasing from a value of $\sim 1 \mathrm{erg} \mathrm{cm}^{-2} \mathrm{~s}^{-1}$ in the isolated case to $\sim 100 \mathrm{erg} \mathrm{cm}^{-2} \mathrm{~s}^{-1}$ in the irradiated case at $R=100 \mathrm{AU}$. The surface density in the irradiated disk is sufficient for the disk midplane to remain effectively shielded from all sources of UV irradiation.

\subsection{Chemical Structure}

In Figure 2, we present the fractional abundance (relative to number density) of gas-phase $\mathrm{CO}, \mathrm{HCO}^{+}, \mathrm{CN}, \mathrm{HCN}, \mathrm{C}_{2} \mathrm{H}, \mathrm{CS}$, $\mathrm{H}_{2} \mathrm{CO}$, and $\mathrm{N}_{2} \mathrm{H}^{+}$as a function of disk radius, $R$, and height, $R / Z$. These species have been detected in numerous nearby gasrich primordial isolated protoplanetary disks due their relatively large abundance and simple rotational spectra leading to strong emission lines (see, e.g., Thi et al. 2004; Dutrey et al. 2007, 2011; Henning et al. 2010; Öberg et al. 2010a). In Figure 3, we present the vertical column densities as a function of disk radius, $R$. We have smoothed the column densities using a Bézier function of index, $n$, where $n$ is the number of data points. We also present the column densities for those additional species observed in protoplanetary disks in absorption and/or emission at infrared wavelengths: $\mathrm{OH}, \mathrm{H}_{2} \mathrm{O}, \mathrm{C}_{2} \mathrm{H}_{2}$, and $\mathrm{CO}_{2}$ (bottom, see, e.g., Lahuis et al. 2006; Carr \& Najita 2008; Mandell et al. 2012). We also show the grain-surface column densities (dashed lines) for species which exhibit significant freezeout. In Table 1, we list the calculated column densities at radii of $10 \mathrm{AU}$ and $100 \mathrm{AU}$

We see a different chemical stratification in the irradiated disk, compared with the isolated disk. The higher dust temperature in the disk midplane allows volatile molecules, such as $\mathrm{CO}$, to remain in the gas, whereas tightly bound molecules, such as $\mathrm{HCN}$, freeze onto grain surfaces. Instead of the usual "warm molecular layer" observed between $0.3 \lesssim Z / R \lesssim 0.5$, we see a thinner molecular layer that is deeper in the disk and abundant in radicals, e.g., $\mathrm{CN}, \mathrm{C}_{2} \mathrm{H}$, and $\mathrm{CS}$, and molecules. $\mathrm{HCO}^{+}$resides in a layer slightly higher than the "molecule/radical" layer 

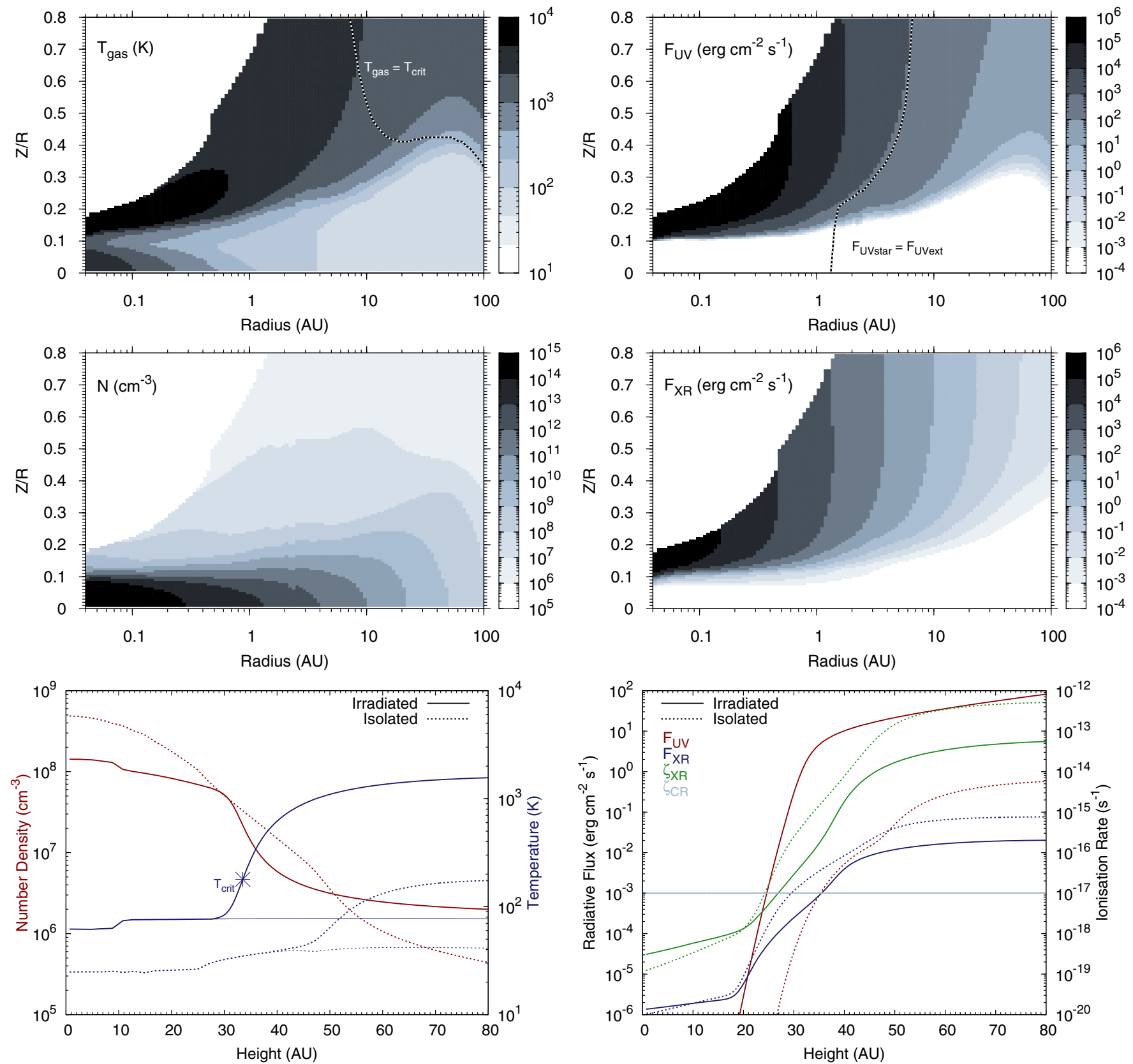

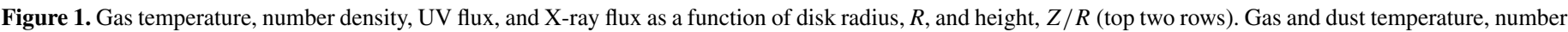

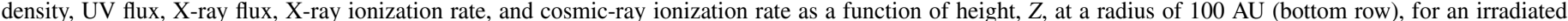

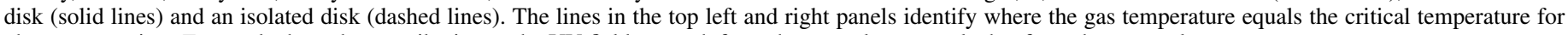
photoevaporation, $T_{\text {crit }}$, and where the contribution to the UV field strength from the central star equals that from the external star.

(A color version of this figure is available in the online journal.)

reflecting the abundance of proton-donating molecules, such as, $\mathrm{H}_{3}{ }^{+}$and $\mathrm{H}_{3} \mathrm{O}^{+}$.

Gas-phase $\mathrm{CO}$ possesses its canonical fractional abundance of $10^{-4}$ throughout the disk midplane and also survives in the disk surface beyond $R \gtrsim 10 \mathrm{AU}$ with a fractional abundance, $\sim 10^{-7}$. Thus, the disk remains significantly molecular in nature, even under extreme irradiation. We see hints of the truncation of the molecular disk beyond $\approx 50 \mathrm{AU}$. Here, we have assumed hydrostatic equilibrium, whereas, in reality, photoevaporative flow may affect the surface density of the outer disk. The disk truncation radius depends primarily on the scale of the flow (see, e.g., Adams et al. 2004).
HCN is depleted in the disk midplane due to freezeout and reaches a fractional abundance of $\sim 10^{-7}$ in the "molecule/radical" layer beyond $R=1 \mathrm{AU}$. Within this radius, thermal desorption from dust grains increases the fractional abundance to $\sim 10^{-4}$. $\mathrm{HCO}^{+}$and $\mathrm{CN}$ both reach a fractional abundance of $\sim 10^{-6}$, whereas $\mathrm{C}_{2} \mathrm{H}$ and $\mathrm{CS}$ reach a value of $\sim 10^{-7} . \mathrm{H}_{2} \mathrm{CO}$ and $\mathrm{N}_{2} \mathrm{H}^{+}$reach lower peak values of $\sim 10^{-8}$ and $\sim 10^{-9}$, respectively. $\mathrm{N}_{2} \mathrm{H}^{+}$reaches this value within a radius $\lesssim 1$ AU only.

Comparing column densities at $10 \mathrm{AU}$, there are minor differences between models (less than a factor of three) for most species. However, in the irradiated disk, the CN, CS, 

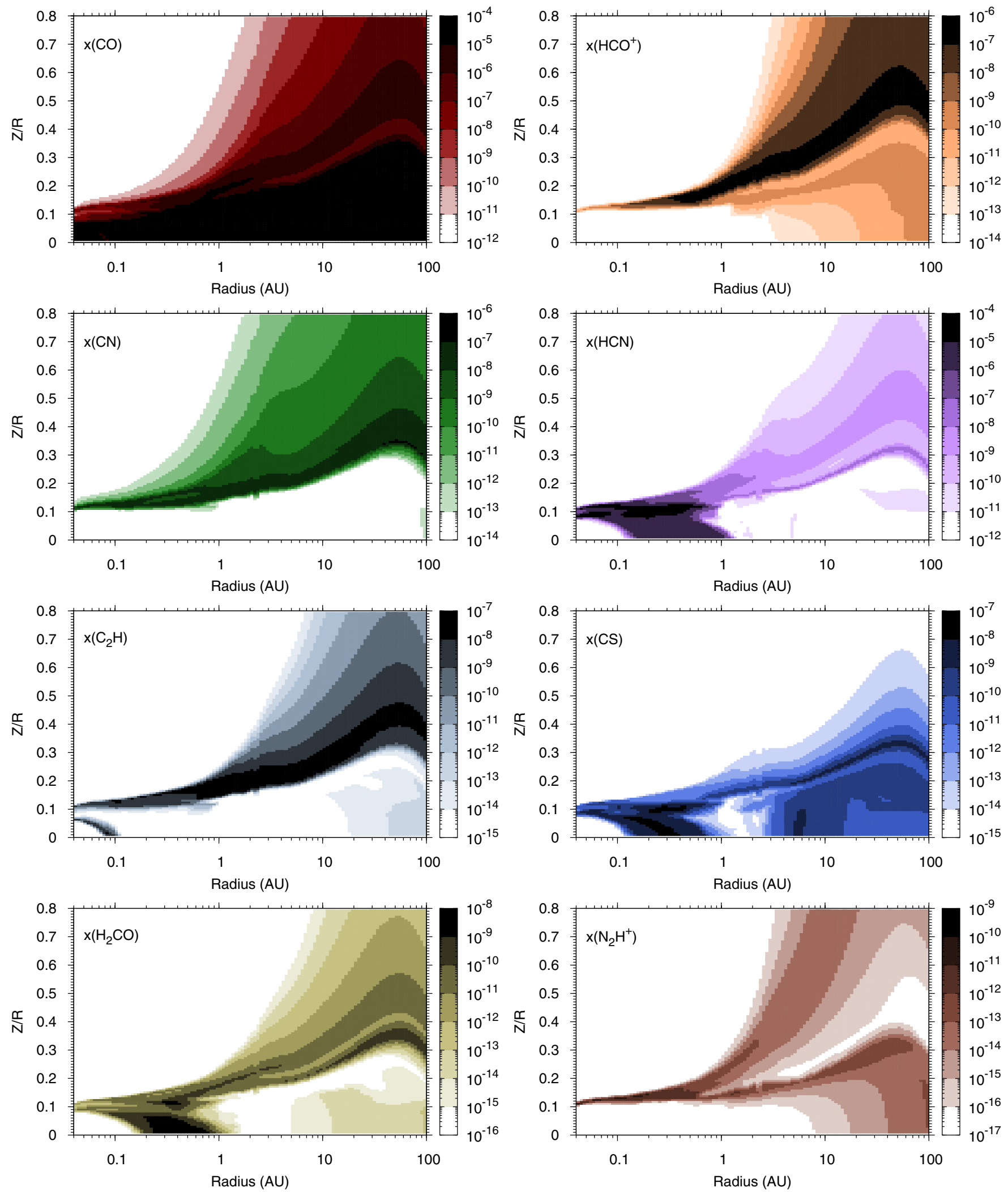

Figure 2. Fractional abundance of molecules (relative to number density) as a function of disk radius, $R$, and height, $Z / R$.

(A color version of this figure is available in the online journal.)

and $\mathrm{CO}_{2}$ column densities are larger, by factors of $4.7,44$, and 1800 , respectively, and those for $\mathrm{N}_{2} \mathrm{H}^{+}, \mathrm{H}_{2} \mathrm{O}$, and $\mathrm{C}_{2} \mathrm{H}_{2}$, are smaller, by factors of 9.6, 7.0, and 9.6, respectively. The $\mathrm{CN} / \mathrm{HCN}, \mathrm{OH} / \mathrm{H}_{2} \mathrm{O}, \mathrm{C}_{2} \mathrm{H} / \mathrm{C}_{2} \mathrm{H}_{2}$ column density ratios are also larger in the irradiated disk, $1.9,2.9$, and 8.2 versus $0.16,0.26$, and 0.29 , respectively, indicative of increased photodissociation of $\mathrm{HCN}, \mathrm{H}_{2} \mathrm{O}$, and $\mathrm{C}_{2} \mathrm{H}_{2}$. Both models exhibit peaks in the CS column density where the dust temperatures are comparable 

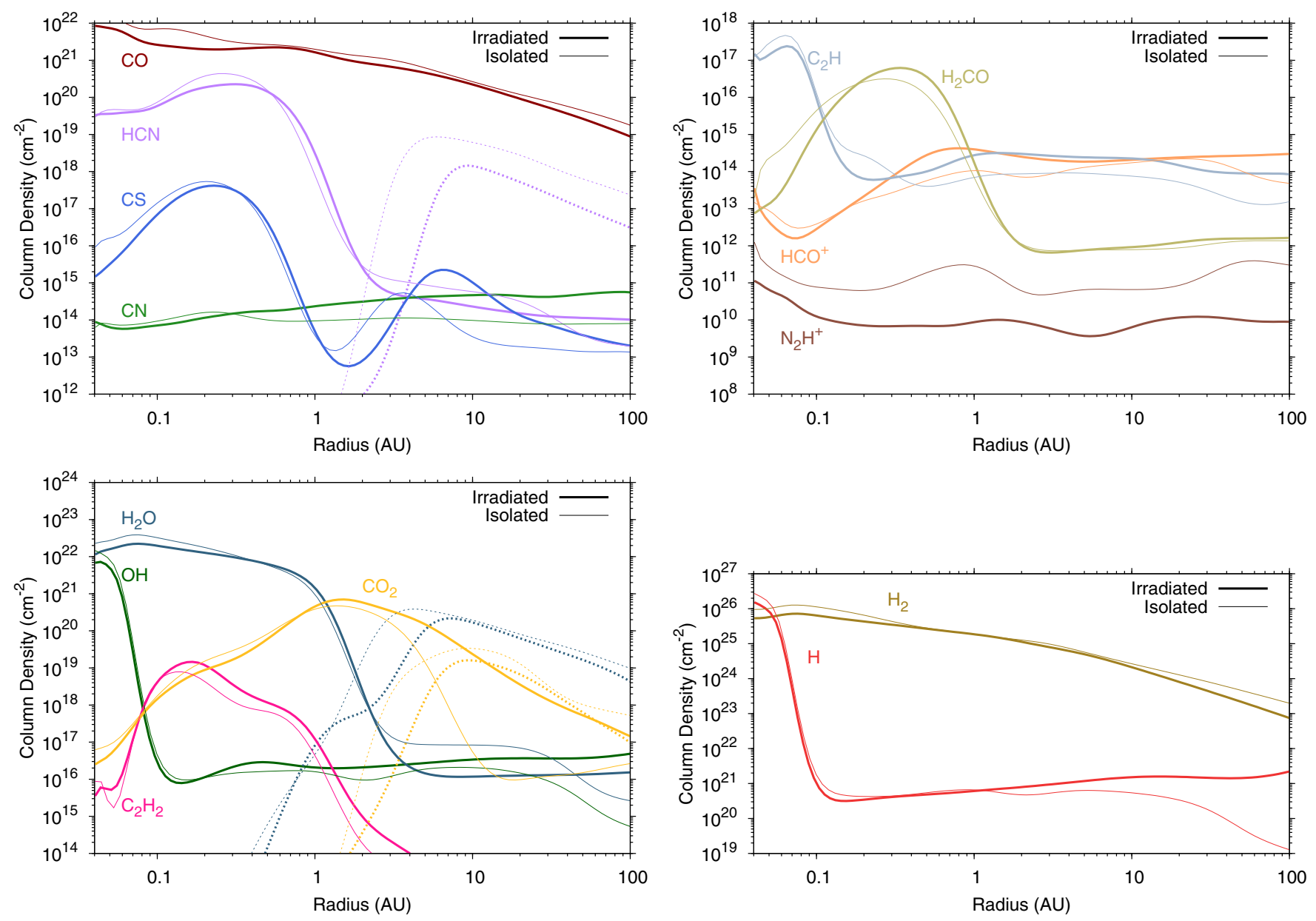

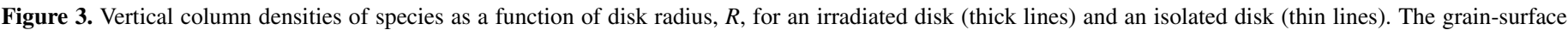
(ice) column densities are represented by the dashed lines.

(A color version of this figure is available in the online journal.)

(10 $\mathrm{AU}$ in the irradiated disk and 4-5 $\mathrm{AU}$ in the isolated disk.) Hence, freezeout onto dust grains accounts for the difference in the CS column densities at $10 \mathrm{AU}$. The $\mathrm{CO}_{2}$ column density is significantly larger in the irradiated disk $\approx 2 \times 10^{19} \mathrm{~cm}^{-2}$ versus $\sim 10^{16} \mathrm{~cm}^{-2}$. In the isolated disk, the snow line for $\mathrm{CO}_{2}$ lies within $10 \mathrm{AU}$; however, in the irradiated disk, the temperature in the midplane at $10 \mathrm{AU}$, and beyond, is high enough for $\mathrm{CO}_{2}$ to possess a significant gas-phase abundance.

At $100 \mathrm{AU}$, the effects of external irradiation are clear. The $\mathrm{H} / \mathrm{H}_{2}$ column density ratio is much larger in the irradiated disk, $2.9 \times 10^{-2}$ versus $6.5 \times 10^{-5}$ for the isolated disk. The $\mathrm{OH} / \mathrm{H}_{2} \mathrm{O}$ and $\mathrm{C}_{2} \mathrm{H} / \mathrm{C}_{2} \mathrm{H}_{2}$ column density ratios are also larger, 3.3 and 7.0 versus 0.20 and 0.47 , respectively. The $\mathrm{OH}$ and $\mathrm{H}_{2} \mathrm{O}$ column densities also increase significantly in the irradiated disk, by factors of 94 and 5.8, respectively. $\mathrm{OH}$ and $\mathrm{H}_{2} \mathrm{O}$ can form efficiently in warm/hot gas $(T \gtrsim 200 \mathrm{~K})$ via the reactions, $\mathrm{H}_{2}+\mathrm{O}$ and $\mathrm{H}_{2}+\mathrm{OH}$ (Glassgold et al. 2009). The $\mathrm{HCO}^{+}$column density is a factor of 6.3 larger in the irradiated disk, tracing the increased ionization degree. We see only minor differences in the column densities of all other species, except $\mathrm{CO}_{2}$ (already discussed) and $\mathrm{N}_{2} \mathrm{H}^{+}$, the latter of which is a factor of 35 smaller in the irradiated disk, at $100 \mathrm{AU} . \mathrm{N}_{2} \mathrm{H}^{+}$is destroyed via reactions with $\mathrm{CO}$ and electrons. The $\mathrm{CO}$ abundance in both models is similar; however, the electron abundance in the molecular layer of the irradiated model is several orders of magnitude higher so that $\mathrm{N}_{2} \mathrm{H}^{+}$destruction via dissociative recombination is more effective in this model.

The best astrophysical analog of our isolated disk model is TW Hya. We achieve good agreement with the disk-averaged abundance ratios for $\mathrm{CN} / \mathrm{HCN}$ derived by Thi et al. (2004; 7.1 versus 3.8 in our model) and the fractional abundance of water vapor determined by Hogerheijde et al. $\left(2011 ; 5-20 \times 10^{-8}\right.$ versus $\left.1-3 \times 10^{-8}\right)$. Adjusting for source size and distance, we achieve reasonable agreement with $\mathrm{H}_{2} \mathrm{CO}$ line strengths observed by Qi et al. (2013): 1.22 and $0.54 \mathrm{Jy} \mathrm{km} \mathrm{s}^{-1}$ versus 0.65 and $0.82 \mathrm{Jy} \mathrm{km} \mathrm{s}^{-1}$ from our model for the $4_{14}-3_{13}$ and $5_{15}-4_{14}$ transitions, respectively (C. Walsh et al., in preparation). We get poor agreement for the $\mathrm{HCO}^{+} / \mathrm{CO}$ ratio from Thi et al. (2004; $1.4 \times 10^{-4}$ versus $2.5 \times 10^{-6}$ ) and our $\mathrm{N}_{2} \mathrm{H}^{+}$line strengths are around an order of magnitude lower than that observed. The truncation of our disk at $100 \mathrm{AU}$ means we do not account for the depletion of $\mathrm{CO}$ onto dust grains at larger radii where $T_{\text {dust }} \lesssim$ $20 \mathrm{~K}$. As discussed previously, $\mathrm{N}_{2} \mathrm{H}^{+}$is destroyed via reaction with $\mathrm{CO}$.

\subsection{Molecular Line Emission}

In Figure 4, we present our synthetic line spectra in ALMA receiver bands $6-9$. We assume a maximum disk radius of $100 \mathrm{AU}$ and a distance to source of $400 \mathrm{pc}$. The lines in bands 

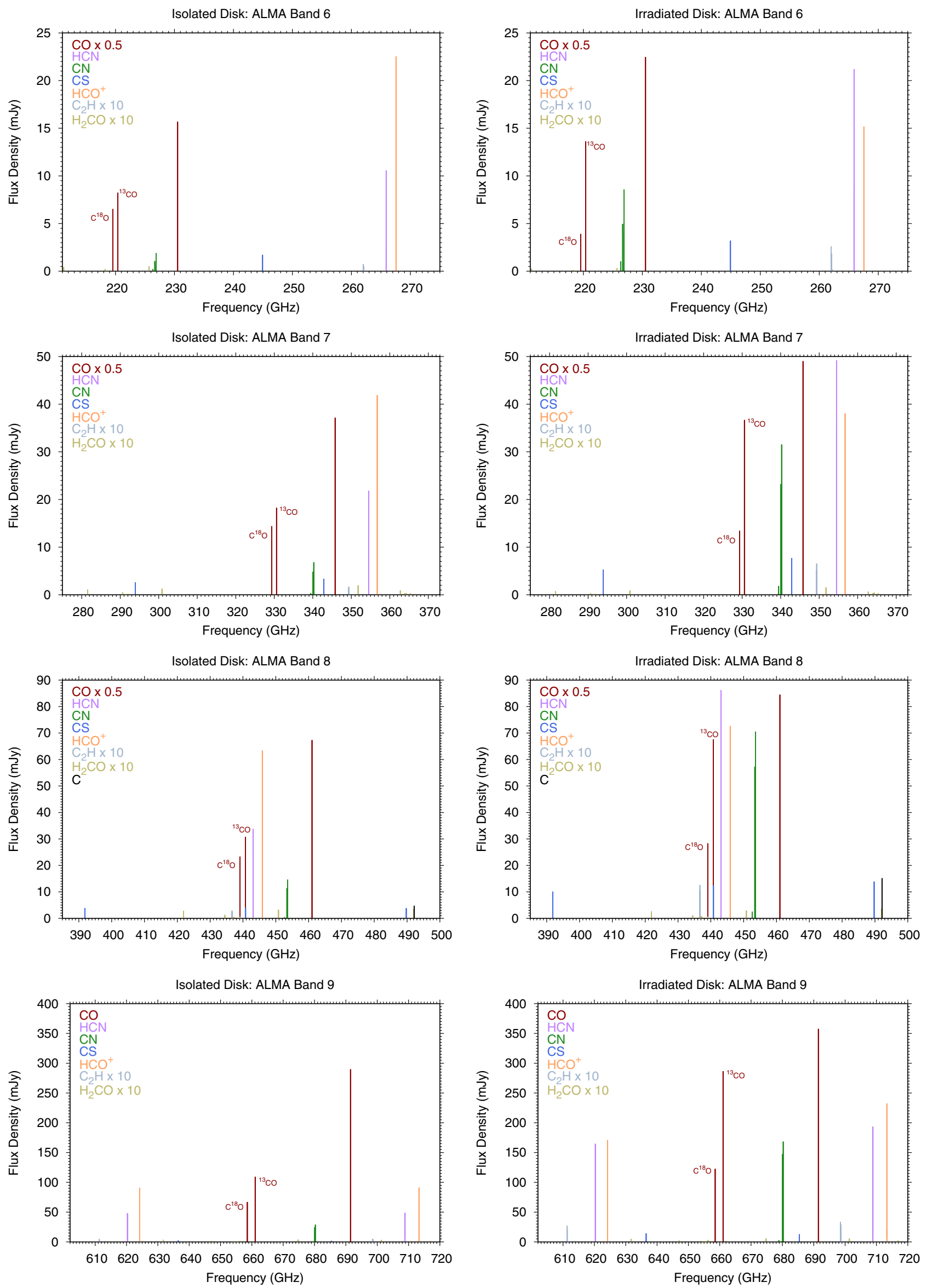

Figure 4. Synthetic spectra in ALMA receiver bands 6-9 for an isolated disk (left) and an irradiated disk (right).

(A color version of this figure is available in the online journal.) 
Table 1

Column Densities and Integrated Line Strengths

\begin{tabular}{|c|c|c|c|c|c|c|c|c|}
\hline \multirow[t]{3}{*}{ Species } & \multicolumn{4}{|c|}{$\begin{array}{l}\text { Column Density } \\
\left(\mathrm{cm}^{-2}\right)\end{array}$} & \multirow[t]{3}{*}{ Transition } & \multirow[t]{3}{*}{$\begin{array}{l}\text { Frequency } \\
\quad(\mathrm{GHz})\end{array}$} & \multicolumn{2}{|c|}{$\begin{array}{l}\text { Line Strength } \\
\left(\mathrm{mJy} \mathrm{km} \mathrm{s}^{-1}\right)\end{array}$} \\
\hline & \multicolumn{2}{|c|}{ Isolated } & \multicolumn{2}{|c|}{ Irradiated } & & & \multirow[t]{2}{*}{ Isolated } & \multirow[t]{2}{*}{ Irradiatec } \\
\hline & $10 \mathrm{AU}$ & $100 \mathrm{AU}$ & $10 \mathrm{AU}$ & $100 \mathrm{AU}$ & & & & \\
\hline $\mathrm{H}$ & $5.6(20)$ & $1.3(19)$ & $1.6(21)$ & $2.2(21)$ & $\ldots$ & $\ldots$ & $\ldots$ & $\ldots$ \\
\hline $\mathrm{H}_{2}$ & $2.6(24)$ & $2.0(23)$ & $2.1(24)$ & $7.5(22)$ & $\ldots$ & $\ldots$ & $\ldots$ & $\ldots$ \\
\hline \multirow[t]{2}{*}{$\mathrm{C}$} & $2.3(17)$ & $7.2(16)$ & $4.7(17)$ & $2.3(17)$ & ${ }^{3} \mathrm{P}_{1}-{ }^{3} \mathrm{P}_{0}$ & 492.161 & $\ldots$ & 22.8 \\
\hline & & & & & ${ }^{3} \mathrm{P}_{2}-{ }^{3} \mathrm{P}_{1}$ & 809.342 & 24.8 & 85.2 \\
\hline \multirow[t]{7}{*}{$\mathrm{CO}$} & $2.7(20)$ & $1.8(19)$ & $2.3(20)$ & $8.8(18)$ & $1-0$ & 115.271 & 14.5 & 31.2 \\
\hline & & & & & $2-1$ & 230.538 & 68.2 & 141 \\
\hline & & & & & $3-2$ & 345.796 & 163 & 326 \\
\hline & & & & & $4-3$ & 461.041 & 293 & 578 \\
\hline & & & & & $6-5$ & 691.473 & 599 & 1230 \\
\hline & & & & & $7-6$ & 806.652 & 734 & 1600 \\
\hline & & & & & $8-7$ & 921.800 & 824 & 1970 \\
\hline \multirow[t]{7}{*}{$\mathrm{HCO}^{+}$} & $1.7(14)$ & $4.8(13)$ & $2.1(14)$ & $3.0(14)$ & $3-2$ & 267.558 & 27.1 & 31.6 \\
\hline & & & & & $4-3$ & 356.734 & 54.1 & 86.9 \\
\hline & & & & & $5-4$ & 445.903 & 86.4 & 184 \\
\hline & & & & & $7-6$ & 624.209 & 150 & 545 \\
\hline & & & & & $8-7$ & 713.342 & 183 & 830 \\
\hline & & & & & $9-8$ & 802.456 & 225 & 1200 \\
\hline & & & & & $10-9$ & 891.558 & 284 & 1670 \\
\hline \multirow[t]{11}{*}{$\mathrm{CN}$} & $9.7(13)$ & $8.0(13)$ & $4.6(14)$ & $5.5(14)$ & $2_{5 / 2}-1_{3 / 2}$ & 226.876 & $\ldots$ & 12.0 \\
\hline & & & & & $3_{5 / 2}-2_{3 / 2}$ & 340.031 & $\ldots$ & 32.9 \\
\hline & & & & & $3_{7 / 2}-2_{5 / 2}$ & 340.249 & $\ldots$ & 45.4 \\
\hline & & & & & $4_{7 / 2}-35 / 2$ & 453.390 & 14.0 & 83.2 \\
\hline & & & & & $4_{9 / 2}-3_{7 / 2}$ & 453.607 & 18.1 & 104 \\
\hline & & & & & $6_{11 / 2}-5_{9 / 2}$ & 680.047 & 32.4 & 221 \\
\hline & & & & & $6_{13 / 2}-5_{11 / 2}$ & 680.264 & 38.2 & 255 \\
\hline & & & & & $7_{13 / 2}-6_{11 / 2}$ & 793.336 & 37.7 & 278 \\
\hline & & & & & $7_{15 / 2}-6_{13 / 2}$ & 793.554 & 43.7 & 315 \\
\hline & & & & & $8_{15 / 2}-7_{13 / 2}$ & 906.593 & 39.5 & 308 \\
\hline & & & & & $8_{17 / 2}-7_{15 / 2}$ & 906.811 & 44.7 & 345 \\
\hline \multirow[t]{7}{*}{$\mathrm{HCN}$} & $5.9(14)$ & $2.1(13)$ & $2.4(14)$ & $1.0(14)$ & $3-2$ & 265.886 & 12.4 & 30.1 \\
\hline & & & & & $4-3$ & 354.505 & 27.1 & 71.9 \\
\hline & & & & & $5-4$ & 443.116 & 44.9 & 129 \\
\hline & & & & & $7-6$ & 620.304 & 80.2 & 251 \\
\hline & & & & & $8-7$ & 708.877 & 98.0 & 295 \\
\hline & & & & & $9-8$ & 797.433 & 120 & 316 \\
\hline & & & & & $10-9$ & 885.971 & 149 & 313 \\
\hline \multirow[t]{7}{*}{ CS } & $2.7(13)$ & $1.5(13)$ & $1.2(15)$ & $2.0(13)$ & $7-6$ & 342.883 & $\ldots$ & 10.8 \\
\hline & & & & & $8-7$ & 391.847 & $\ldots$ & 14.2 \\
\hline & & & & & $9-8$ & 440.803 & $\ldots$ & 17.3 \\
\hline & & & & & $10-9$ & 489.751 & $\ldots$ & 19.6 \\
\hline & & & & & $13-12$ & 636.532 & $\ldots$ & 20.2 \\
\hline & & & & & $14-13$ & 685.436 & $\ldots$ & 18.5 \\
\hline & & & & & $17-16$ & 832.062 & $\ldots$ & 11.3 \\
\hline $\mathrm{C}_{2} \mathrm{H}$ & $7.7(13)$ & $1.4(13)$ & $2.3(14)$ & $8.4(13)$ & $\ldots$ & $\ldots$ & $\ldots$ & $\ldots$ \\
\hline $\mathrm{H}_{2} \mathrm{CO}$ & $8.1(11)$ & $1.4(12)$ & $9.3(11)$ & $1.6(12)$ & $\ldots$ & $\ldots$ & $\ldots$ & $\ldots$ \\
\hline $\mathrm{N}_{2} \mathrm{H}^{+}$ & $6.7(10)$ & $3.1(11)$ & 7.0(09) & $8.9(09)$ & $\ldots$ & $\ldots$ & $\ldots$ & $\ldots$ \\
\hline $\mathrm{H}_{2} \mathrm{O}$ & $8.4(16)$ & $2.6(15)$ & $1.2(16)$ & $1.5(16)$ & $\ldots$ & $\ldots$ & $\ldots$ & $\ldots$ \\
\hline $\mathrm{OH}$ & $2.2(16)$ & $5.2(14)$ & $3.5(16)$ & $4.9(16)$ & $\ldots$ & $\ldots$ & $\ldots$ & $\ldots$ \\
\hline $\mathrm{CO}_{2}$ & $1.3(16)$ & $2.7(16)$ & $2.4(19)$ & $1.4(17)$ & $\ldots$ & $\ldots$ & $\ldots$ & $\ldots$ \\
\hline $\mathrm{C}_{2} \mathrm{H}_{2}$ & $2.7(14)$ & $3.0(13)$ & $2.8(13)$ & $1.2(13)$ & $\ldots$ & $\ldots$ & $\ldots$ & $\ldots$ \\
\hline
\end{tabular}

Notes.

a $a(b)=a \times 10^{b}$.

${ }^{\mathrm{b}}$ Listed line strengths are restricted to $>10 \mathrm{mJy} \mathrm{km}^{-1}$.

3 and 4 are likely too weak to be observable and observations in band 10 are difficult due to increased atmospheric absorption at higher frequencies. Due primarily to the higher gas temperature, the emission lines from the irradiated disk reach higher peak values than the equivalent lines from the isolated disk. This is most notable for $\mathrm{C}, \mathrm{CO}$ (and isotopologues), $\mathrm{CN}, \mathrm{CS}$, and
$\mathrm{HCN}$. The $\mathrm{HCO}^{+}$transitions in bands 6 and 7 reach higher peak flux densities in the isolated disk and are also much stronger than those for $\mathrm{HCN}$, despite both molecules possessing similar rotational spectra. $\mathrm{HCO}^{+}$is a tracer of cold, dense material and is generally more abundant than $\mathrm{HCN}$ in the outer disk midplane. 
In Table 1, we list the transitions, frequencies, and integrated line intensities. Due to the higher gas temperature in the irradiated disk $(\gg 50 \mathrm{~K})$, lower frequency transitions $(v \sim 100 \mathrm{GHz})$ are weaker than higher frequency transitions and the integrated line strengths are stronger than those for the isolated disk. A significant proportion of the $\mathrm{CO},{ }^{13} \mathrm{CO}$, and $\mathrm{C}^{18} \mathrm{O}$ ladders should be observable with ALMA advantageously allowing characterization of the gas excitation temperature, column density, and optical depth (see, e.g., Bruderer et al. 2012). Multiple transitions of $\mathrm{CI}, \mathrm{HCO}^{+}, \mathrm{HCN}$, and $\mathrm{CN}$ may also be observable allowing additional characterization of the physical conditions. The $\mathrm{CN} / \mathrm{HCN}$ and $\mathrm{CI} / \mathrm{CO}$ line ratios and derived column densities would also allow a measurement of the degree of photodestruction in the disk. The remaining molecules would be difficult to observe; however, $\mathrm{CS}$ and $\mathrm{C}_{2} \mathrm{H}$ may be observable in larger,

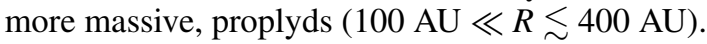

\section{DISCUSSION}

Proplyds have been extensively studied since their discovery (see, e.g., O’Dell et al. 1993; Bally et al. 1998), and were originally observed in silhouette against the bright nebular background. Early models concentrated on characterizing physical properties: morphology, surface density, mass, dust distribution, mass-loss rates, and lifetime (see, e.g., Johnstone et al. 1998; Störzer \& Hollenbach 1999; Richling \& Yorke 2000; Adams et al. 2004; Clarke 2007). Models generally agree that the disk survives for a few Myr, beyond which photoevaporation destroys the disk on a timescale $\sim 10^{5} \mathrm{yr}$, depending on the distance from the massive star. In Figure 1, we identify the region where the gas temperature equals the critical temperature for photoevaporation, i.e., where the photoevaporative flow likely originates. According to Dullemond et al. (2007), $T_{\text {crit }} \approx 0.2 T_{\text {virial }}$, where $T_{\text {virial }}$ is the temperature at which the sound speed equals the escape velocity of the gas. Several groups (e.g., Adams et al. 2004) model the disk as a photondominated region (PDR) including a small chemical network for the computation of the thermal balance and do not report the molecular structure beyond the transition from atomic to molecular hydrogen. Other authors report chemical structure calculations and resulting line emission; however, this has been restricted to ionic and atomic lines (see, e.g., Richling \& Yorke 2000). Nguyen et al. (2002) studied the chemistry in irradiated disks around high-mass and low-mass stars, modeling the disk structure as a series of one-dimensional vertical PDRs. They categorize "observable" molecules as those possessing a value $\geqslant 10^{12} \mathrm{~cm}^{-2}$. In their low-mass model, their column densities for $\mathrm{HCN}, \mathrm{H}_{2} \mathrm{CO}, \mathrm{H}_{2} \mathrm{O}$, and $\mathrm{C}_{2} \mathrm{H}$ are larger than ours by more than an order of magnitude despite both models generating similar $\mathrm{CO}$ column densities. We find the opposite case for $\mathrm{HCO}^{+}$ and $\mathrm{CN}$. These differences likely arise from different prescriptions for the disk structure: we perform a self-consistent calculation of the physical structure, whereas Nguyen et al. (2002) adopt assumed conditions. Our work highlights the importance of radiative transfer calculations in determining the potential observability of molecules, rather than relying on column density calculations.

Here, we report the results of a self-consistent twodimensional model of the physical and chemical structure of a protoplanetary disk irradiated by a nearby massive star. The disk remains predominantly molecular in nature over the lifetime of the disk $\left(\approx 10^{6} \mathrm{yr}\right)$ with potentially observable abundances of atoms and molecules $\left(\mathrm{CI}, \mathrm{CO}, \mathrm{HCO}^{+}, \mathrm{CN}\right.$, and $\left.\mathrm{HCN}\right)$ at (sub)mm wavelengths. The disk midplane is effectively shielded from UV radiation such that non-volatile molecules, e.g., $\mathrm{HCN}$ and $\mathrm{H}_{2} \mathrm{O}$, are frozen out onto dust grains suggesting icy planetesimal formation may be possible in proplyds. Observations of multiple transitions of these species (and isotopologues) would allow characterization of the gas surface density, excitation temperature, and optical depth. As far as we are aware, the work reported here is the first attempt to model the two-dimensional molecular structure and resultant (sub)mm molecular line emission of a proplyd.

Astrophysics at QUB is supported by a grant from the STFC. H. Nomura acknowledges the Grant-in-Aid for Scientific Research 21740137, 23103005 and the Global COE Program "The Next Generation of Physics, Spun from Universality and Emergence" from MEXT, Japan.

\section{REFERENCES}

Adams, F. C. 2010, ARA\&A, 48, 47

Adams, F. C., Hollenbach, D., Laughlin, G., \& Gorti, U. 2004, ApJ, 611, 360

Allamandola, L. J., Sandford, S. A., \& Valero, G. J. 1988, Icar, 76, 225

Bally, J., Sutherland, R. S., Devine, D., \& Johnstone, D. 1998, AJ, 116, 293

Bruderer, S., van Dishoeck, E. F., Doty, S. D., \& Herczeg, G. J. 2012, A\&A, 541, A91

Carr, J. S., \& Najita, J. R. 2008, Sci, 319, 1504

Ciaravella, A., Mũnoz Caro, G., Jiménez Escobar, A., et al. 2010, ApJL, 722, L45

Clarke, C. J. 2007, MNRAS, 376, 1350

D’Alessio, P., Calvet, N., Hartmann, L., Franco-Hernández, R., \& Servín, H. 2006, ApJ, 638, 314

D’Hendecourt, L. B., Allamandola, L. J., Grim., R. J. A., \& Greenberg, J. M. 1986, A\&A, 158, 119

Dullemond, C. P., \& Dominik, C. 2004, A\&A, 421, 1075

Dullemond, C. P., Hollenbach, D., Kamp, I., \& D'Alessio, P. 2007, in Protostars and Planets V, ed. B. Reipurth, D. Jewitt, \& K. Keil (Tucson, AZ: Univ. Arizona Press), 555

Dutrey, A., Henning, T., Guilloteau, S., et al. 2007, A\&A, 464, 615

Dutrey, A., Wakelam, V., Boehler, Y., et al. 2011, A\&A, 535, A104

Glassgold, A. E., Meijerink, R., \& Najita, J. R. 2009, ApJ, 701, 142

Hagen, W., Allamandola, L. J., \& Greenberg, J. M. 1979, Ap\&SS, 65, 215

Hasegawa, T. I., \& Herbst, E. 1993, MNRAS, 261, 83

Hasegawa, T. I., Herbst, E., \& Leung, C. M. 1992, ApJS, 82, 167

Henning, Th., Semenov, D., Guilloteau, St., et al. 2010, ApJ, 714, 1511

Hogerheijde, M. R., Bergin, E. A., Brinch, C., et al. 2011, Sci, 334, 338

Johnstone, D., Hollenbach, D., \& Bally, J. 1998, ApJ, 499, 758

Kennicutt, R. C., Jr., \& Evans, N. J., II 2012, ARA\&A, 50, 531

Lahuis, F., van Dishoeck, E. F., Boogert, A. C. A., et al. 2006, ApJL, 636, L145

Mandell, A. M., Bast, J., van Dishoeck, E. F., et al. 2012, ApJ, 747, 92

Nomura, H., Aikawa, Y., Tsujimoto, M., Nakagawa, Y., \& Millar, T. J. 2007, ApJ, 661,334

Nomura, H., \& Millar, T. J. 2005, A\&A, 438, 923

Nguyen, T. K., Viti, S., \& Williams, D. A. 2002, A\&A, 387, 1083

Öberg, K. I., Linnartz, H., Visser, R., \& van Dishoeck, E. F. 2009a, ApJ, 693, 1209

Öberg, K. I., Qi, C., \& Fogel, J. K. J. 2010a, ApJ, 720, 480

Öberg, K. I., van Dishoeck, E. F., \& Linnartz, H. 2009b, A\&A, 496, 281

Öberg, K. I., van Dishoeck, E. F., Linnartz, H., \& Andersson, S. 2010b, ApJ, 718,832

O’Dell, C. R., Wen, Z., \& Hu, X. 1993, ApJ, 410, 696

Pringle, J. E. 1981, ARA\&A, 19, 137

Qi, C., Öberg, K. I., \& Wilner, D. J. 2013, ApJ, 765, 34

Richling, S., \& Yorke, H. W. 2000, ApJ, 539, 258

Ruffle, D. P., \& Herbst, E. 2001, MNRAS, 322, 770

Störzer, H., \& Hollenbach, D. 1999, ApJ, 515, 669

Thi, W.-F., van Zadelhoff, G.-J., \& van Dishoeck, E. F. 2004, A\&A, 425, 955

Walsh, C., Millar, T. J., \& Nomura, H. 2010, ApJ, 722, 1607

Walsh, C., Nomura, H., Millar, T. J., \& Aikawa, Y. 2012, ApJ, 747, 114

Weingartner, J. C., \& Draine, B. T. 2001, ApJ, 548, 296

Wilson, T. L., \& Rood, R. T. 1994, ARA\&A, 32, 191

Woodall, J., Agúndez, M., Markwick-Kemper, A. J., \& Millar, T. J. 2007, A\&A, 466,1197 